\title{
Disfunción eréctil durante el tratamiento con isotretinoína
}

\author{
A. Tirado Sánchez, G. León Dorantes \\ Servicio de Dermatología. Hospital General de México.
}

Actas Urol Esp 2005; 29 (10): 974-976

\section{RESUMEN}

DISFUNCIÓN ERÉCTIL DURANTE EL TRATAMIENTO CON ISOTRETINOÍNA

La isotretinoína es un medicamento muy efectivo para el manejo del acné severo resistente al tratamiento convencional. Sin embargo, su uso se encuentra asociado con numerosos efectos secundarios, algunos de ellos de índole psiquiátrica. Durante la realización de un estudio prospectivo para evaluar la eficacia y seguridad de la isotretinoína en pacientes adultos con acné severo, seis pacientes hombres, refirieron dificultades para mantener una adecuada erección del pene asociado a datos clínicos de depresión. En nuestro conocimiento ésta es la primera vez que se reporta este efecto colateral, así que los resultados sugieren que se deben realizar más estudios de su uso, investigando específicamente este efecto secundario.

Palabras clave: Disfunción eréctil. Isotretinoína. Depresión.

\section{ABSTRACT}

\section{ERECTILE DYSFUNCTION DURING ISOTRETINOIN THERAPY}

Isotretinoin is a very effective drug for the treatment of severe recalcitrant acne. However, it's use is associated with many side effects, sometimes is associated with the emergence of psychiatric symptoms. During of a prospective study to evaluate the efficacy and safety of isotretinoin in acne, six male patients, refer difficulties in maintaining adequate penile erection in association with clinical symptoms of depression. To our knowledge this is the first time that this side effect is reported, so the results suggest that further studies of it's use in similar patients are warranted.

Keywords: Erectile dysfunction. Isotretinoin. Depression.

$\mathrm{L}$ a isotretinoína es un medicamento efectivo en el manejo del acné severo resistente al tratamiento convencional con antibióticos orales. Sin embargo, su uso se ha asociado a efectos colaterales sistémicos, algunos de los cuales pueden llegar a ser muy serios. Uno de los efectos más severos es la presencia de síntomas psiquiátricos (depresión moderada a severa); no obstante tal relación hasta este momento es controvertida ${ }^{1,2}$.

La disfunción eréctil, que se puede relacionar a depresión severa, no la encontramos documentada en asociación con consumo de isotretinoína. A continuación presentamos dicho efecto asociado a pacientes en tratamiento de entre 3 y 4 meses con isotretinoína, recalcando que es la primera vez que se documenta.

\section{MATERIAL Y MÉTODO}

Durante la realización de un estudio prospectivo para evaluar la eficacia y seguridad de la isotretinoína en acné severo en pacientes adultos, uno de los pacientes tratado con el medicamento, a los 3 meses reportó la presencia de dificultad para iniciar y mantener la erección del pene, situación que anteriormente refería no haber presentado. Por dicho motivo iniciamos un estudio de los mismos pacientes del estudio enfocado a la detección de disfunción eréctil y su relación con la isotretinoína y síntomas depresivos.

En el estudio original contábamos con 20 pacientes del sexo masculino con acné que recibieron isotretinoína entre 3 y 4 meses y 35 pacien- 
tes del grupo control que recibieron tratamiento con minociclina por el mismo tiempo. Para los fines de este nuevo estudio sobre el desarrollo de la disfunción eréctil, se consideró el grupo de la isotretinoína como grupo de investigación (con 20 casos) y el de minociclina como control (35 pacientes).

Además de una nueva exploración física e interrogatorio dirigido (sobre todo presencia de tabaquismo, hipertensión y diabetes), se les aplicó la Escala de Hamilton para Depresión (EHD) y el Índice Internacional de 5 puntos para Disfunción Eréctil (IIDE). Dentro de los estudios de laboratorio solicitados figuraban: colesterol total, niveles séricos de testosterona y sulfato de dehidroepiandrostenediona.

Se decidió eliminar a estos pacientes del estudio original y se continuó valoración de manera independiente para determinar persistencia de la disfunción eréctil una vez suspendido el tratamiento.

\section{ANÁLISIS ESTADÍSTICO}

Para el estudio descriptivo usamos medidas de tendencia central y de dispersión. Para el estudio comparativo usamos una prueba de $\mathrm{X}^{2} \mathrm{y}$ la prueba exacta de Fisher, mientras que para determinar el riesgo que implicaba el consumo de isotretinoína para desarrollar disfunción eréctil, realizamos un análisis univariado con intervalo de confianza al 95\%, considerando como estadísticamente significativo un valor de $p<0,05$. Utilizamos el paquete estadístico SPSS versión 10 para Windows (Chicago, Ill).

\section{RESULTADOS}

Se reclutaron 55 pacientes de los cuáles 20 correspondian a pacientes que tomaron isotretinoína y 35 controles que recibieron tratamiento con minociclina. La media de la edad de los casos era de 32,6 años, mientras que los controles tenían una media de edad de 33,5 años, sin encontrar diferencias estadísticamente significativas $(p=<0,05)$. Todos los pacientes estudiados se encontraban entre los 3 y 4 meses de tratamiento .

Seis de los 20 pacientes (30\%) del grupo de casos presentaron disfunción eréctil, mientras que sólo 2 de los 35 pacientes (5,7\%) del grupo control presentaron esta alteración (se catalogó como disfunción eréctil en base a los valores obtenidos del IIDE donde se obtuvieron puntuaciones de 10 o menos para cada paciente. Puntuaciones de $21 \mathrm{o}$ inferiores son indicativos de disfunción eréctil). Ninguno de los pacientes con la alteración refería haber presentado disfunción eréctil con anterioridad y además presentaban puntuaciones elevadas en la escala de Hamilton, por lo que es posible que la disfunción eréctil se desarrollara al inicio de los síntomas depresivos.

La determinación de algunos estudios de laboratorio como colesterol total, niveles séricos de testosterona y sulfato de dehidroepiandrostenediona, presentaron valores dentro de límites normales.

Se decidió eliminar a estos pacientes del estudio original y se continuó valoración de manera independiente, con conducta expectante para determinar persistencia de la disfunción eréctil una vez suspendido el tratamiento. A los 4 a 6 meses de descontinuado el medicamento, observamos una marcada reducción de los valores de la EHD y un incremento en los resultados del IIDE (que se midieron mensualmente), traduciéndose clínicamente en una remisión de los síntomas de depresión, con restablecimiento de la función eréctil del pene a la normalidad.

Al estudio comparativo encontramos que sí existe diferencia en la presencia de disfunción eréctil en relación al tipo de tratamiento instaurado, presentándose más en el grupo de tratamiento con isotretinoína, al igual que la presencia de depresión moderada a severa (Tabla 1).

$\mathrm{Al}$ análisis univariado, el tratamiento con isotretinoína se comporta como un factor de riesgo para disfunción eréctil, incrementando el riesgo hasta 6 veces, mientras que la presencia de depresión incrementa el riesgo 2 veces en comparación con los pacientes que no la presentan (Tabla 2).

\section{DISCUSIÓN}

Es posible que la isotretinoína, que puede conducir a depresión a través de los receptores de retinoides en el sistema nervioso central ${ }^{1}$, se relacione de manera indirecta con la disfunción eréctil a través de la depresión con la que se asocia y es probable que al inducir hipercolesterolemia, esto pueda contribuir en el efecto secundario $^{4}$, aunque en nuestros casos los pacientes tenían niveles de colesterol sérico normales. 
Tabla 1

Estudio comparativo de las variables estudiadas

\begin{tabular}{lccc}
\hline Variable & $\begin{array}{c}\text { Casos } \\
(\mathbf{n = 2 0})\end{array}$ & $\begin{array}{c}\text { Controles } \\
(\mathbf{n}=\mathbf{3 5})\end{array}$ & $\begin{array}{c}\text { Valor } \\
\text { de } \boldsymbol{p}\end{array}$ \\
\hline Disfunción eréctil & 6 & 2 & $0,021^{*}$ \\
Tabaquismo (+) & 11 & 15 & $\mathrm{SDE}$ \\
Hipertensión arterial (+) & 3 & 5 & $\mathrm{SDE}$ \\
$\begin{array}{l}\text { Escala de Hamilton } \\
\text { (depresión moderada }\end{array}$ & 12 & 3 & 0,039 \\
$\begin{array}{l}\text { a severa) } \\
\text { Hipercolesterolemia (+) }\end{array}$ & 5 & 7 & $\mathrm{SDE}$ \\
Testosterona $(<437 \mathrm{ng} / \mathrm{dl})$ & 2 & 2 & $\mathrm{SDE}$ \\
DHEA** $(<800 \mathrm{mcg} / \mathrm{l})$ & 2 & 2 & $\mathrm{SDE}$ \\
\hline
\end{tabular}

Prueba de Ji cuadrada. *Prueba exacta de Fisher.

**DHEA.- Dehidroepiandrosterona. SDE.- Sin diferencia estadística.

Tabla 2

Influencia del tratamiento con isotretinoína y de la depresión moderada a severa en la disfunción eréctil.

\begin{tabular}{lccc}
\hline Variable & $\mathbf{R M}$ & IC $^{95 \%}$ & Valor de $\boldsymbol{p}$ \\
Isotretinoína & 7,071 & $1,2-39,4$ & 0,021 \\
$\begin{array}{l}\text { Depresión moderada } \\
\text { a severa }\end{array}$ & 3,273 & $1-10,4$ & 0,039 \\
\hline
\end{tabular}

Análisis Univariado (Ji cuadrada)

RM.- Razón de Momios

$\mathrm{IC}_{95 \%}$ - Intervalo de confianza al 95\%

El acné por sí mismo puede relacionarse con depresión moderada a severa y teóricamente puede producir también disfunción eréctil ${ }^{5}$.

Recomendamos la realización de manera rutinaria de la EHD y del IIDE en pacientes masculinos con vida sexual activa en tratamiento con isotretinoína, por ser pruebas de fácil realización y que permiten detectar casos de disfunción eréctil, para poder suspender con anticipación la administración del medicamento (isotretinoína) y buscar otras opciones de tratamiento del acné y de realizar, en caso necesario valoración psiquiátrica y/o urológica.

No obstante, son necesarios estudios controlados al respecto para poder determinar si lo observado con la isotretinoína y la disfunción eréctil es causa-efecto, o simplemente casualidad.

\section{REFERENCIAS}

1. Hull PR, D’Arcy C. Isotretinoin use and subsequent depression and suicide: presenting the evidence. Am J Clin Dermatol 2003;4(7):493-505.

2. Ferahbas A, Turan MT, Esel E, Utas S, Kutlugun C, Kilic CG. A pilot study evaluating anxiety and depressive scores in acne patients treated with isotretinoin. J Dermatolog Treat. 2004; 15(3):153-157.

3. Rosen RC, Cappelleri JC, Smith MD, Lipsky J, Pena BM. Development and evaluation of an abridged, 5-item version of the International Index of Erectile Function (IIEF-5) as a diagnostic tool for erectile dysfunction. Int $\mathrm{J}$ Impot Res 1999;11(6):319-326.

4. Hafez ES, Hafez SD. Erectile dysfunction: anatomical parameters, etiology, diagnosis, and therapy. Arch Androl 2005; 51(1):15-31.

5. Tan JK. Psychosocial impact of acne vulgaris: evaluating the evidence. Skin Therapy Lett 2004;9(7):1-3.

Dr. A. Tirado Sánchez

Servicio de Dermatología

Hospital General de México

Dr. Balmis, 148

Col. Roma Sur, Mexico D.F.

06720 D.F. México

E-mail: andrestiradosanchez@hotmail.com

(Trabajo recibido el 19 de mayo de 2005) 